\title{
Suppression of the expression of hypoxia-inducible factor-1 $\alpha$ by RNA interference alleviates hypoxia-induced pulmonary hypertension in adult rats
}

\author{
YING LI, BO SHI, LIPING HUANG, XIN WANG, XIAONA YU, BAOSHENG GUO and WEIDONG REN
}

Department of Ultrasound Medicine, Shengjing Hospital of China Medical University, Shenyang, Liaoning 110004, P.R. China

Received December 11, 2015; Accepted October 5, 2016

DOI: 10.3892/ijmm.2016.2773

\begin{abstract}
Hypoxia-inducible factor-1 $\alpha$ (HIF-1 $\alpha)$ has been implicated in the pathogenesis of hypoxic pulmonary hypertension (PH). However, the potential clinical value of HIF-1 $\alpha$ as a therapeutic target in the treatment of PH has not yet been evaluated. In this study, an animal model of hypoxia-induced $\mathrm{PH}$ was established by exposing adult rats to $10 \% \mathrm{O}_{2}$ for 3 weeks, and the effects of the lentivirus-mediated delivery of HIF-1 $\alpha$ short hairpin RNA (shRNA) by intratracheal instillation prior to exposure to hypoxia on the manifestations of hypoxia-induced PH were assessed. The successful delivery of HIF- $1 \alpha$ shRNA into the pulmonary arteries effectively suppressed the hypoxiainduced upregulation of HIF-1 $\alpha$, accompanied by the prominent attenuation the symptoms associated with hypoxia-induced $\mathrm{PH}$, including the elevation of pulmonary arterial pressure, hypertrophy and hyperplasia of pulmonary artery smooth muscle cells (PASMCs), as well as the muscularization of pulmonary arterioles. In addition, the knockdown of HIF-1 $\alpha$ in cultured rat primary PASMCs significantly inhibited the hypoxia-induced acceleration of the cell cycle and the proliferation of the PASMCs, suggesting that HIF-1 $\alpha$ may be a direct mediator of PASMC hyperplasia in hypoxia-induced PH. In conclusion, this study demonstrates the potent suppressive effects of HIF- $1 \alpha$ shRNA on hypoxia-induced $\mathrm{PH}$ and PASMC hyperplasia, providing evidence for the potential application of HIF-1 $\alpha$ shRNA in the treatment of hypoxic $\mathrm{PH}$.
\end{abstract}

\section{Introduction}

Pulmonary hypertension (PH) is a complication of numerous pulmonary conditions. $\mathrm{PH}$ is currently classified into five categories according to different etiologies of the disease as follows: pulmonary arterial hypertension, $\mathrm{PH}$ due to left heart disease,

Correspondence to: Dr Weidong Ren, Department of Ultrasound Medicine, Shengjing Hospital of China Medical University, 36 Sanhao Street, Shenyang, Liaoning 110004, P.R. China

E-mail: renwd01@163.com

Key words: hypoxia-inducible factor- $1 \alpha$, pulmonary hypertension, RNA interference, pulmonary artery smooth muscle cells
$\mathrm{PH}$ due to lung disease, chronic thromboembolic $\mathrm{PH}$ and $\mathrm{PH}$ due to unclear multifactorial mechanisms (1). If left untreated, $\mathrm{PH}$ can lead to right-sided heart failure and even mortality $(2,3)$.

Prolonged exposure to alveolar hypoxia, at a high altitude or secondary to chronic pulmonary or cardiovascular diseases, is an important cause of PH (4). Acute hypoxia induces vasoconstriction that causes a reversible increase in pulmonary vascular resistance, whereas prolonged hypoxia stimulates vascular remodeling and persistent vasoconstriction in hypoxic PH by inducing the proliferation and migration of pulmonary arterial smooth muscle cells (PASMCs), altering the behavior of pulmonary arterial endothelial cells (PAECs) and myofibroblasts, and accumulating the extracellular matrix proteins secreted by the resident cells (5). Moreover, the interactions between different types of cells are also critical in the pathophysiology of PH. For example, PAECs have been shown to release a variety of factors that stimulate PASMC proliferation (6,7). A prominent pathological change in the blood vessels of patients with $\mathrm{PH}$ is the muscularization of distal pulmonary arteries, as evidenced by an in increase in the number of $\alpha$-smooth muscle actin ( $\alpha$-SMA)-positive cells in in situ immunohistochemical analysis (8), implying that PASMCs act as an essential player in the pathogenesis of $\mathrm{PH}$.

Hypoxia-inducible factor-1 (HIF-1) is a nuclear transcription factor that functions as a master regulator of adaptive responses to hypoxia (9). HIF-1 is a heterodimer composed of an $\mathrm{O}_{2}$-regulated HIF-1 $\alpha$ subunit and a constitutively expressed HIF-1 $\beta$ subunit. Under hypoxic conditions, HIF-1 is stabilized and activates the transcription of numerous genes, the products of which are involved in angiogenesis, erythropoiesis, energy metabolism and cell survival (10). HIF-1 has been implicated in the pathogenesis of $\mathrm{PH}$ based on both experimental and clinical data. Mice with heterozygous knockout of HIF-1 $\alpha\left(\mathrm{Hifla}^{+/-}\right)$ have been shown to display attenuated manifestations of chronic hypoxia-induced $\mathrm{PH}$, such as hypoxia-induced muscularization of pulmonary arterioles and medial wall thickening (11). Recently, using a mouse model with smooth muscle-specific knockout of HIF-1 $\alpha$, Ball et al demonstrated that HIF-1 $\alpha$ in the smooth muscle cells played a crucial role in pulmonary vascular remodeling and the development of $\mathrm{PH}$ in response to chronic hypoxia (12). In addition, clinical studies have revealed a marked elevation in HIF-1 $\alpha$ expression in proliferating PAECs of plexiform lesions within the lungs of patients with severe PH (13). 
These findings implicate HIF-1 $\alpha$ in the pathologic alterations in both PASMCs and PAECs in PH, suggesting that HIF-1 $\alpha$, as a master regulator of hypoxic responses, is a promising therapeutic candidate for hypoxic PH. In addition to HIF-1 $\alpha$, a study on HIF- $2 \alpha$, a HIF-1 $\alpha$ homologue, demonstrated that the heterozygous deletion of HIF-2 $\alpha$ attenuated hypoxia-induced PH in mice (14), suggesting that HIF-2 $\alpha$ also contributes to the development of hypoxic PH; however, whether HIF-3 is involved in the pathogenesis of $\mathrm{PH}$ remains unknown.

In this study, an animal model of hypoxia-induced $\mathrm{PH}$ was established by exposing adult rats to $10 \% \mathrm{O}_{2}$ for 3 weeks. The effects of the lentivirus-mediated delivery of HIF-1 $\alpha$ short hairpin RNA (shRNA), which was administered by intratracheal instillation prior to exposure to hypoxia, on the manifestations of hypoxia-induced $\mathrm{PH}$ were assessed. In addition, rat primary PASMCs were cultured in vitro and transduced with HIF-1 $\alpha$ shRNA in order to examine the anti-proliferative effects of HIF-1 $\alpha$ shRNA on PASMCs.

\section{Materials and methods}

Lentiviruses. An EGFP-encoding lentiviral strains carrying the shRNA oligonucleotides that target 5'-GACCAACAA CUUGAAGAUG-3' or 5'-GCAGCAGGAATTGGAACGT-3' on HIF-1 $\alpha$ mRNA, namely HIF-1 $\alpha$ shRNA \#1 and HIF-1 $\alpha$ shRNA \#2, respectively, were ordered from HanBio (Shanghai, China). A scramble shRNA with the sequence of 5'-GATCCCC GTTCTCCGAACGTGTCACGTTTCAAGAGAACGTGA CACGTTCGGAGAACTTTTT-3' was cloned in parallel to create a control lentiviral strain.

Animal model of chronic hypoxia-induced PH and delivery of lentiviruses to the lungs. All animal experimental procedures were approved by the Animal Care and Ethics Committee of China Medical University, Shenyang, China and performed in accordance with the Guidelines for the Care and Use of Laboratory Animals. The development of the animal model of chronic hypoxia-induced $\mathrm{PH}$ and shRNA delivery to the pulmonary vessels were conducted based on a previously described method (15). Briefly, 6-8-week-old Sprague-Dawley (SD) rats, purchased from Liaoning Changsheng Biotech Biotech Co., Ltd. (Benxi, China), were assigned to the following 5 groups (n=6/group): i) normoxia (untreated); ii) hypoxia; iii) hypoxia + scramble; iv) hypoxia + HIF-1 $\alpha$ shRNA \#1; and v) hypoxia + HIF-1 $\alpha$ shRNA \#2. Following anesthesia, $1.5 \times 10^{8} \mathrm{U}$ of lentiviruses harboring HIF-1 $\alpha$ shRNA or scramble shRNA was administered to each rat by intratracheal instillation through the mouth (in a total of $300 \mu \mathrm{l}$, and $50 \mu \mathrm{l}$ was instilled per day for 6 days). Following 6 days of lentiviral treatment, the rats, apart from those in the normoxia group, were exposed to hypoxia $\left(10 \% \mathrm{O}_{2}\right.$ environment) for 3 weeks to induce $\mathrm{PH}$, and the rats in the normoxia group were maintained under the normoxic conditions $\left(21 \% \mathrm{O}_{2}\right)$ during the same period.

Determination of right ventricular systolic pressure. Following 3 weeks of exposure to hypoxia or normoxia, right ventricular systolic pressure (RVSP) was measured based on a previously described method (16) using the BL420S animal performance analyzing system (Thaimeng, Chengdu, China). Briefly, the rats were anesthetized and placed on their backs. An incision was made on the neck to expose the trachea, which was then connected to a respirator. The respiratory parameters of the mechanical ventilation were set as follows: respiratory rate, $80 \mathrm{breaths} / \mathrm{min}$; tidal volume, $6-10 \mathrm{ml} / \mathrm{kg} / \mathrm{min}$. A catheter was punched in the right external jugular vein and pushed along the vein to reach the right ventricle. The other end of the catheter was connected to the BL420S system for the measurement of RVSP.

Fluorescence microscopy for the determination of lentiviral transduction to the lungs. Following the determination of RVSP, the rats were euthanized by an overdose of anesthesia (10\% chloral hydrate at $5 \mathrm{ml} / \mathrm{kg}$ body weight), and the lungs were excised. A fraction of the lung tissues was fixed with $4 \%$ paraformaldehyde, dehydrated with sucrose and embedded in optimal cutting temperature (OCT) compound, and $10-\mu \mathrm{m}$-thick cryosections were obtained using the Leica CM1860 cryosectioning station (Leica, Wetzlar, Germany). The intensity of green fluorescence protein (GFP) in the lung sections was examined under an Olympus BX53 fluorescence microscope (Olympus, Tokyo, Japan) at x200 magnification.

Isolation of pulmonary arteries from SD rats. The isolation of the pulmonary arteries was performed as previously described (17). Briefly, the rats were sacrificed, and the lungs were excised from the chest cavity and rinsed with phosphate-buffered saline (PBS). The superficial tissue and the bronchus artery were discarded with fine micro-scissors, and the adventitia was removed from the isolated arteries under a dissecting microscope (AE31; Motic Electric, Xiamen, China). The isolated arteries were then frozen in liquid nitrogen for later experiments.

Reverse transcription-quantitative polymerase chain reaction $(R T-q P C R)$. Total RNA was extracted from the rat pulmonary arteries or the cultured PASMCs using the RNAsimple total RNA kit (Tiangen, Beijing, China), and then reverse transcribed into cDNA using Super M-MLV reverse transcriptase (BioTeke, Beijing, China). The mRNA levels of HIF- $1 \alpha$ and $\beta$-actin were determined by quantitative PCR (qPCR) in an Exicycler 96 quantitative fluorescence analyzer (Bioneer, Daejeon, Korea) using SYBR-Green Master Mix (Solarbio, Beijing, China) and the following primers: HIF-1 $\alpha$ forward, 5'-CCTACT ATGTCGCTTTCTTGG-3', and HIF-1 $\alpha$ reverse, 5'-GTTTCT GCTGCCTTGTATGG-3'; $\beta$-actin forward, 5'-GGAGA TTACTGCCCTGGCTCCTAGC-3', and $\beta$-actin reverse, 5'-GGCCGGACTCATCGTACTCCTGCTT-3'.

Western blot analysis. For total protein extraction, the pulmonary arteries were physically homogenized and lysed with RIPA lysis buffer containing $1 \%$ v/v PMSF, and the cultured PASMCs were lysed with NP-40 lysis buffer (both from Beyotime, Haimen, China). Proteins were separated by sodium dodecyl sulfate-polyacrylamide gel electrophoresis (SDS-PAGE), and transferred onto a PVDF membrane (Millipore, Bedford, MA, USA). The membrane was blocked with $5 \%$ milk and incubated with anti-HIF-1 $\alpha$ antibody (1:400; Cat. no. PB0245; Boster, Wuhan, China) overnight at $4^{\circ} \mathrm{C}$, followed by incubation with horseradish peroxidase (HRP)-conjugated goat anti-rabbit IgG secondary antibody (1:5000; Cat. no. A0208; Beyotime) for $45 \mathrm{~min}$ at room temperature. Thereafter, the immune complexes were visualized using the ECL system (Qihai Biotechnology, 
Shanghai, China). To verify equal loading and transfer, the membrane was re-probed with anti- $\beta$-actin antibody $(1: 1000$; Cat.no. sc-47778; Santa Cruz Biotechnology, Inc., Dallas, TX, USA) and goat anti-mouse IgG (1:5000; Cat. no. A0216; Beyotime). The film was scanned and analyzed using Gel-Pro-Analyzer software for densitometric measurement of the target bands using $\beta$-actin as an internal control.

Morphometric and immunohistochemical analyses. A fraction of the excised lungs was fixed in $4 \%$ paraformaldehyde and embedded in paraffin. Paraffin blocks of the lung tissues were sliced into 5- $\mu \mathrm{m}$-thick sections. Some sections were stained with hematoxylin and eosin (H\&E) following the standard protocol for morphological examination as in previous studies (18), and vascular remodeling was assessed based on a previously described method (15). Briefly, 60-80 intra-acinar vessels per rat accompanying either alveolar ducts or alveoli were evaluated by two researchers in a blinded manner. The external diameter and internal diameter of these arteries were measured by the use of ImageJ software (NIH, Bethesda, MD, USA). The arterial wall thickness was calculated as follows: percentage wall thickness $=[($ external diameter - internal diameter)/external diameter] x100.

For immunohistochemical analysis, following antigen retrieval, the lung sections were treated with $3 \% \mathrm{H}_{2} \mathrm{O}_{2}$, blocked with goat serum and incubated with anti- $\alpha$-SMA antibody (Abcam, Cambridge, MA, USA) overnight at $4^{\circ} \mathrm{C}$, followed by serial incubations with biotin-conjugated goat anti-mouse IgG secondary antibody and HRP-labeled streptavidin (both from Beyotime). Diaminobezidine (Sigma-Aldrich, St. Louis, MO, USA) was added to initiate the chromogenic reaction, and hematoxylin was used to stain the nuclei. The sections were mounted and examined under a microscope (DP73; Olympus) at x400 magnification.

Preparation and culture of PASMCs, lentiviral transduction and exposure to hypoxia. Another group of 6-8-week-old healthy male SD rats (purchased from Liaoning Changsheng Biotech Biotech Co., Ltd.) was used for the isolation of PASMCs by an explant method as previously described (19). Briefly, the thoracic aorta was removed and washed with PBS. A longitudinal incision was made, and the inner endothelial layer was scraped off gently with a scalpel. The aorta was cut into 1-3 $\mathrm{mm}^{2}$ pieces. The aortic fragments from 2 rats were placed with the inner surface down in one FBS-rinsed $35 \mathrm{~mm}^{2}$ culture dish. After $12 \mathrm{~h}$ of incubation at $37^{\circ} \mathrm{C}$, approximately $5 \times 10^{5}$ attached cells were obtained. The cells were cultured in Dulbecco's modified Eagle's medium (DMEM) (Gibco, Carlsbad, CA, USA) supplemented with $20 \%$ fetal bovine serum (FBS; HyClone, Logan, UT, USA) at $37^{\circ} \mathrm{C}$ in an atmosphere of $21 \% \mathrm{O}_{2}$ and $5 \% \mathrm{CO}_{2}$. The PASMCs were identified by immunofluorescence staining with anti- $\alpha$-SMA antibody (1:50), and the images were captured under an Olympus BX53 fluorescence microscope at x600 magnification. Cells between passage 3 and 8 were infected with lentiviruses harboring HIF- $1 \alpha$ shRNA \#1 or scramble shRNA, and cultured at $37^{\circ} \mathrm{C}$ in a normoxic environment for $24 \mathrm{~h}$. Thereafter, the medium was changed to fresh culture medium and the cells were placed in a $37^{\circ} \mathrm{C}$ chamber of $5 \% \mathrm{O}_{2}$ and $5 \% \mathrm{CO}_{2}$ for exposure to hypoxia for $48 \mathrm{~h}$. Non-transduced cells were cultured in a normoxic environment $\left(21 \% \mathrm{O}_{2}\right)$ or in a hypoxic environment $\left(5 \% \mathrm{O}_{2}\right)$ as the controls.

3-(4,5-Dimethylthiazol-2-yl)-2,5-diphenyltetrazolium bromide (MTT) cell proliferation assay. At $24 \mathrm{~h}$ after viral infection, the PASMCs were seeded into 96 -well plates at a density of 3,000 cells/well, and the cells were exposed to hypoxia or normoxia for $48 \mathrm{~h}$. Thereafter, the cells were incubated with $0.2 \mathrm{mg} / \mathrm{ml} \mathrm{MTT} \mathrm{(Sigma-Aldrich)} \mathrm{for} 4 \mathrm{~h}$ at $37^{\circ} \mathrm{C}$ in a normoxic environment. Subsequently, the medium was carefully aspirated and $200 \mu \mathrm{l}$ DMSO was added in to each well to dissolve the MTT-formazan crystals completely. Optical density (OD) values at $490 \mathrm{~nm}$ were measured using an ELX-800 microplate reader (BioTek, Winooski, VT, USA). This experiment was performed in 5 replicates.

Flow cytometry for cell cycle analysis. Following viral transduction and $48 \mathrm{~h}$ of exposure to hypoxia or normoxia, the cell cycle of the PASMCs was analyzed by flow cytometry. The cells were fixed in $70 \%$ ethanol for $2 \mathrm{~h}$ at $4^{\circ} \mathrm{C}$, and incubated in propidium iodide (PI) solution containing $50 \mu \mathrm{g} / \mathrm{ml}$ RNase A (Beyotime) for $30 \mathrm{~min}$, followed by analysis using a FACSCalibur flow cytometer (BD Biosciences, Franklin Lakes, NJ, USA).

Statistical analysis. Raw data were analyzed using GraphPad PRISM software (version 5.0; GraphPad Software, San Diego, CA, USA). Values are expressed as the means \pm standard deviation (SD) of individual rats within the group or of 3 independent experiments for the in vitro assays. Comparison between multiple groups was performed using one-way analysis of variance (ANOVA), followed by Bonferroni post-hoc test for comparisons between 2 groups. The difference was considered statistically significant when $\mathrm{P}<0.05$.

\section{Results}

Reduction of hypoxia-induced HIF-1 $\alpha$ expression in pulmonary arteries by RNA interference (RNAi). The inhibition efficiency of HIF- $1 \alpha$ shRNA \#1 and HIF- $1 \alpha$ shRNA \#2 on HIF-1 $\alpha$ expression was tested in an in vitro pilot experiment (data not shown). The EGFP-encoding lentiviruses harboring HIF-1 $\alpha$ shRNA \#1 or HIF-1 $\alpha$ shRNA \#2 were administered into the lungs of the SD rats by intratracheal instillation, and the signals of GFP were predominantly detected in the cells aligning the pulmonary arteries and arterioles 3 weeks after lentiviral administration (Fig. 1A). This probably occurred as the lentivirus underwent replication in the proliferating PAECs and PASMCs in response to chronic hypoxia, and thus only the proliferating cells could produce adequate GFP for microscopic detection. Following exposure to hypoxia for 3 weeks, the expression of HIF-1 $\alpha$ in the pulmonary arteries was increased by 3.59 -fold at the mRNA level $(\mathrm{P}<0.001)$ and by 3.43 -fold at the protein level $(\mathrm{P}<0.001)$, as compared to the normoxia group (Fig. 1B and C). By contrast, both HIF-1 $\alpha$ shRNA \#1 and HIF-1 $\alpha$ shRNA \#2 effectively reduced the hypoxia-induced elevation of HIF-1 $\alpha$ at the mRNA and protein level $(\mathrm{P}<0.001)$ in the pulmonary arteries, whereas the scramble shRNA had no effect on HIF-1 $\alpha$ expression. These results indicated a successful lentivirus-mediated gene delivery 
A
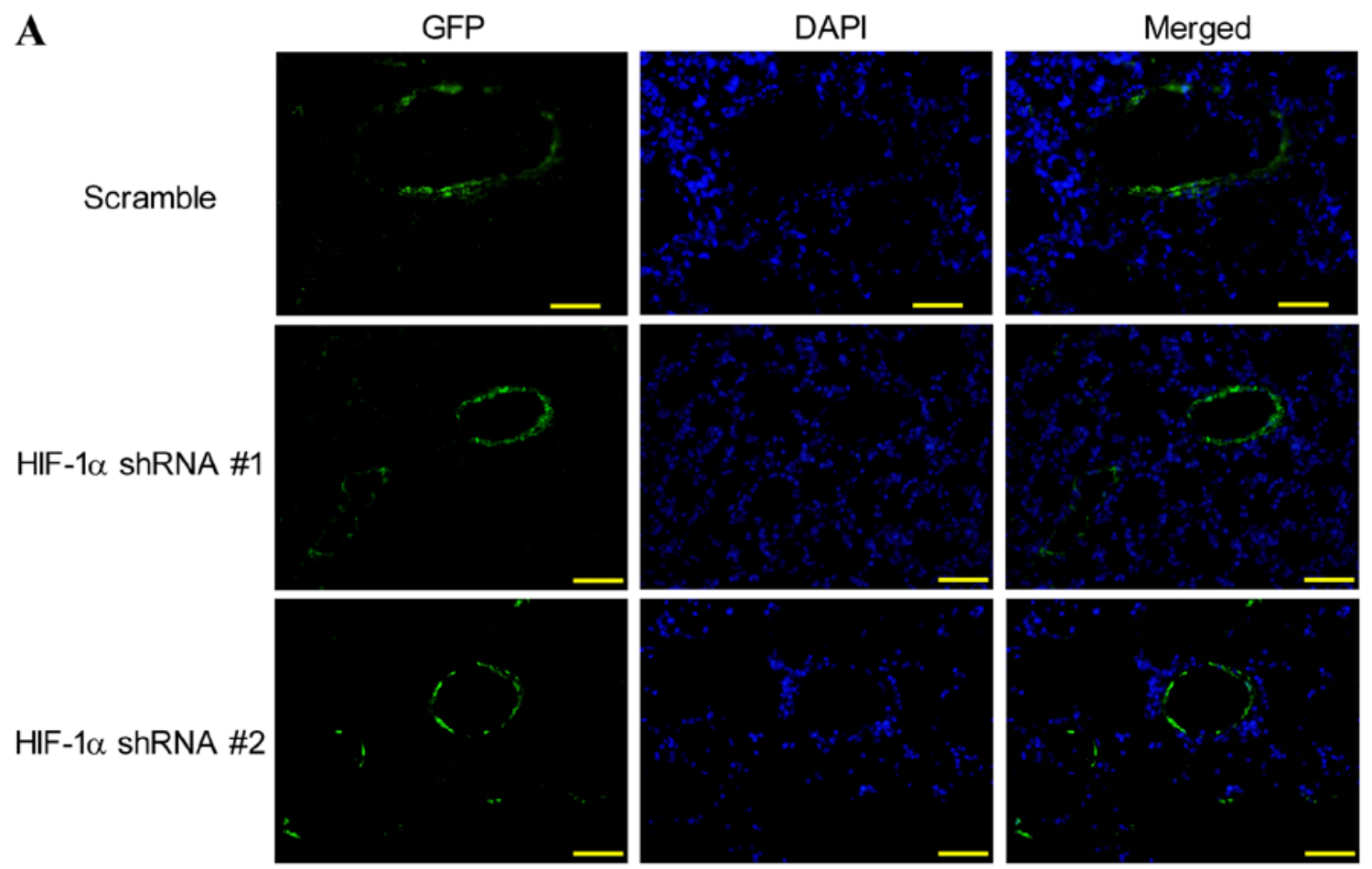

B
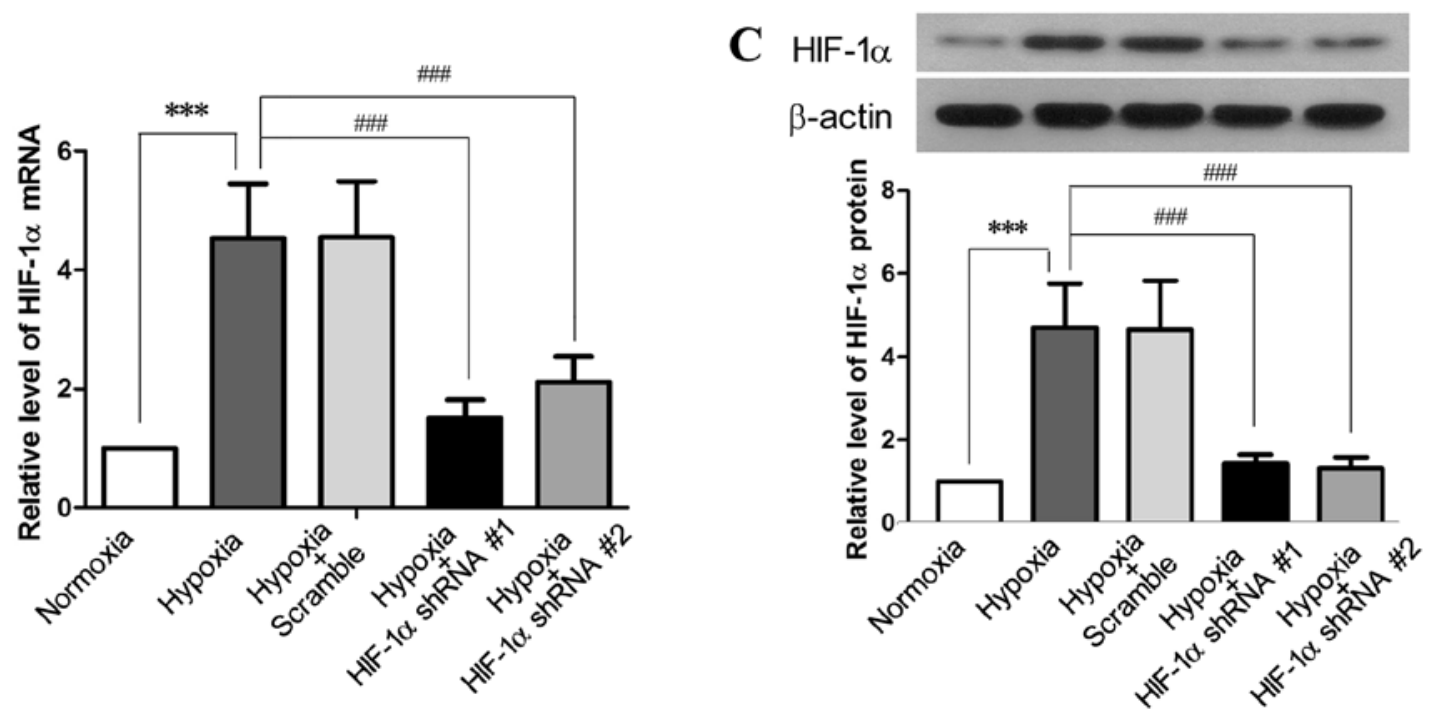

Figure 1. Lentivirus-mediated delivery of hypoxia-inducible factor-1 $\alpha$ (HIF-1 $\alpha$ ) shRNA to pulmonary arteries inhibits the hypoxia-induced upregulation of HIF-1 $\alpha$. SD rats received EGFP-encoding lentiviruses carrying HIF-1 $\alpha$ shRNA \#1, HIF-1 $\alpha$ shRNA \#2 or scramble shRNA by intratracheal instillation for 6 days, followed by exposure to hypoxia $\left(10 \% \mathrm{O}_{2}\right)$ for 3 weeks. SD rats without lentiviral treatment were exposed to hypoxia or normoxia for 3 weeks as the control (n=6 for each group). (A) Following exposure to hypoxia, the cryosections of the lungs from the rats receiving lentiviral treatment were prepared and examined under a fluorescence microscope (x200 magnification; scale bar, $100 \mu \mathrm{m}$ ) to detect the expression of green fluorescence protein (GFP), which was primarily aligned with the pulmonary vessels. Following lentiviral treatment and exposure to hypoxia, the rats were sacrificed and the pulmonary arteries were isolated. The levels of (B) HIF-1 $\alpha$ mRNA and (C) HIF-1 $\alpha$ protein in the pulmonary arteries were assessed by RT-qPCR and western blot analysis, respectively. This figure shows the representative images from each group, and the values are expressed as the means $\pm \mathrm{SD}$. ${ }^{* * *} \mathrm{P}<0.001$, hypoxia vs. normoxia; ${ }^{\# \# \prime} \mathrm{P}<0.001$, hypoxia + HIF-1 $\alpha$ shRNAs vs. hypoxia.

to the pulmonary arteries and a marked inhibition efficiency of the HIF-1 $\alpha$ shRNAs against the hypoxia-induced upregulation of HIF-1 $\alpha$.

Suppression of the expression of HIF-1 $\alpha$ in pulmonary arteries attenuates hypoxia-induced $\mathrm{PH}$ and pulmonary arterial remodeling. The morphology of the lung vessels following exposure to chronic hypoxia with and without manipulation of HIF-1 $\alpha$ expression was examined by H\&E staining. The results revealed an increased thickness of the pulmonary arterioles due to smooth muscle cell (SMC) hypertrophy (increase in cell volume) and hyperplasia (over-proliferation) (Fig. 2A), which are hallmarks of $\mathrm{PH}$ (20). Morphometric analysis revealed that chronic hypoxia increased the medial thickness of the pulmonary arterioles compared with the normoxic controls (36 \pm 8.2 vs. $18.9 \pm 5.2 \% ; \mathrm{P}<0.001)$, and HIF- $1 \alpha$ shRNA \#1 significantly attenuated the hypoxia-induced vascular remodeling and the thickening of the pulmonary arterioles $(\mathrm{P}<0.05$; Fig. $2 \mathrm{~B})$. 
A

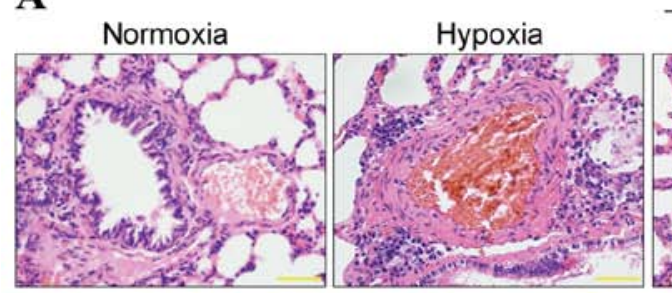

B

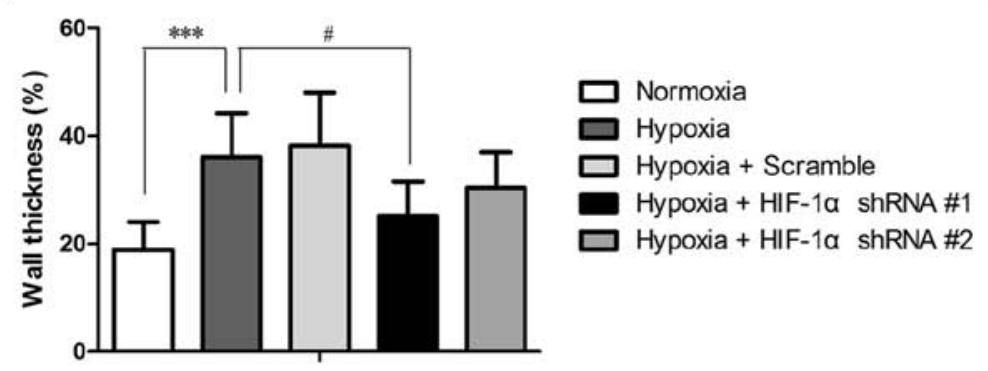

D
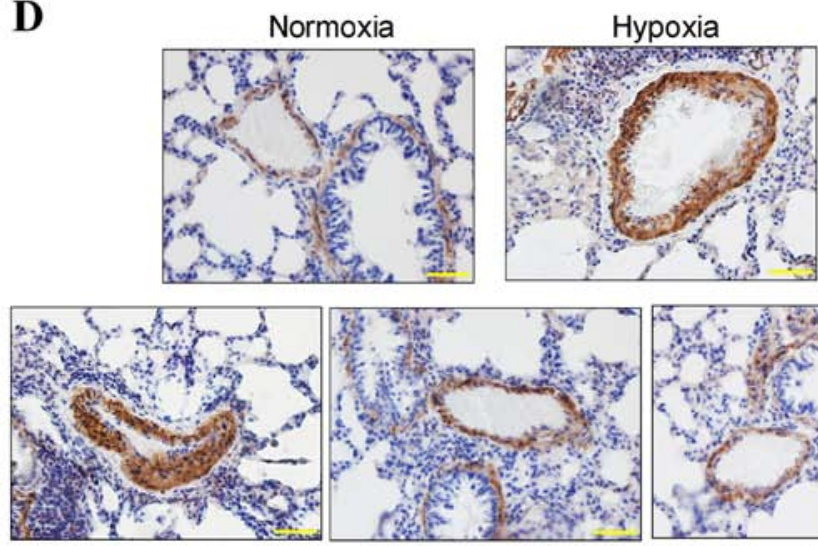

Scramble

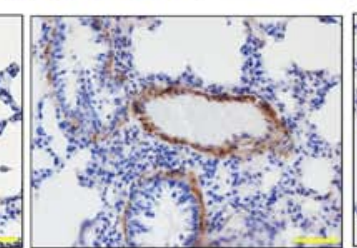

HIF- $1 \alpha$ shRNA \#1

Hypoxia

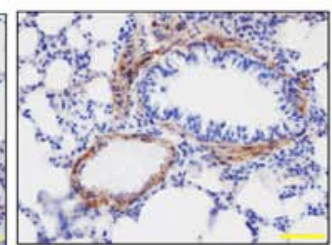

HIF-1 $\alpha$ shRNA \#2
Hypoxia

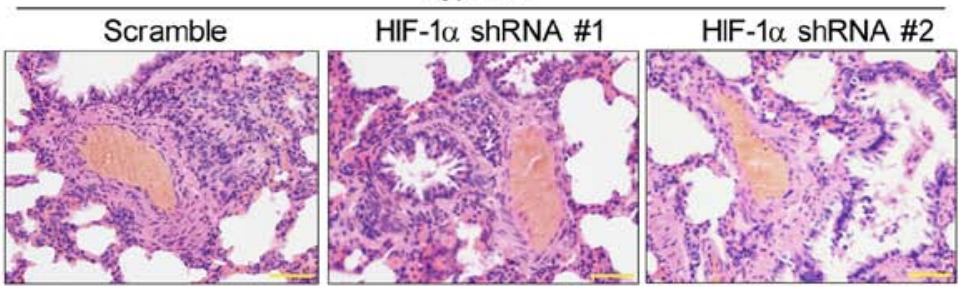

C

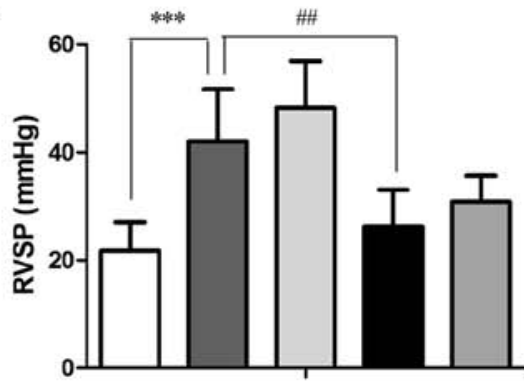

E

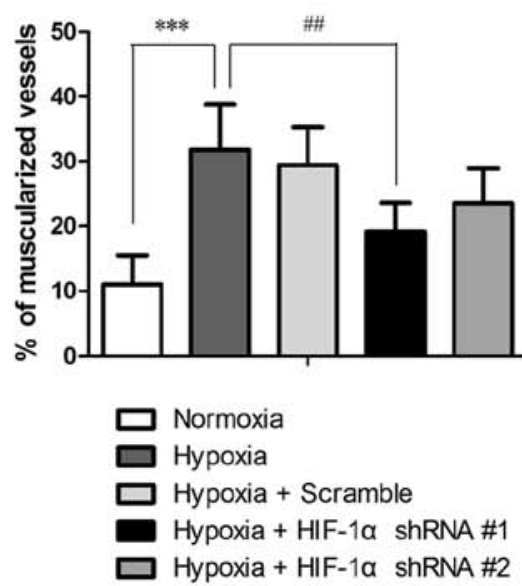

Figure 2. Hypoxia-inducible factor-1 $\alpha$ (HIF-1 $\alpha$ ) shRNA pre-treatment reduced hypoxia-induced pulmonary hypertension (PH) and pulmonary arterial remodeling. SD rats with and without pre-treatment of HIF-1 $\alpha$ shRNA were exposed to hypoxia for 3 weeks. (A) Hematoxylin and eosin (H\&E) staining of the lung sections were performed, and the sections were examined microscopically under a 400X lens (scale bar, $50 \mu \mathrm{m}$ ). (B) The external diameter and internal diameter of the arteries were measured based on H\&E images using ImageJ software, and the percentage wall thickness was calculated as described in the Materials and methods. (C) Following exposure to hypoxia or normoxia, right ventricular systolic pressure (RVSP) was measured. (D) The degree of muscularization of pulmonary arteries was assessed by immunohistochemical staining for $\alpha$-smooth muscle actin ( $\alpha$-SMA) in the lung sections. The images were photographed at $\mathrm{x} 400$ magnification (scale bar, $50 \mu \mathrm{m}$ ), and (E) the percentage of fully muscularized vessels was statistically analyzed based on 60-80 vessels/rat. The figure shows the representative images from each group ( $\mathrm{n}=6$ /group), and the values are expressed as the means $\pm \mathrm{SD}$. ${ }^{* * *} \mathrm{P}<0.001$, hypoxia vs. normoxia;; $\mathrm{P}<0.05$ and ${ }^{\# \#} \mathrm{P}<0.01$, hypoxia + HIF-1 $\alpha$ shRNAs vs. hypoxia.

However, the reduction in hypoxia-induced vessel wall thickening by HIF-1 $\alpha$ shRNA \#2 lacked statistical significance, which was probably due to the small sample size.

RVSP, an indicator of pulmonary arterial pressure, was measured in the rats from all groups ( $\mathrm{n}=6$ each group). RVSP in rats under normoxics conditions was $21.73 \pm 5.3 \mathrm{mmHg}$, and the rats that were exposed to $10 \% \mathrm{O}_{2}$ for 3 weeks developed $\mathrm{PH}$ with an RVSP of $42.04 \pm 9.7 \mathrm{mmHg}$, which was significantly higher than that of the normoxic controls ( $\mathrm{P}<0.01$; Fig. 2C). By contrast, the RVSP of the rats that were pre-treated with HIF- $1 \alpha$ shRNA was $26.19 \pm 6.9$ and $30.83 \pm 4.82 \mathrm{mmHg}$ for HIF- $1 \alpha$ shRNA \#1 and HIF-1 $\alpha$ shRNA \#2, respectively. The RVSP values of the rats that were pre-treated with HIF-1 $\alpha$ shRNA were markedly reduced compared to those of the hypoxia group $(\mathrm{P}<0.05)$, and no statistically significant differences were observed compared to the normoxia group, suggesting that the rats treated with HIF-1 $\alpha$ shRNA did not develop PH after 3 weeks of exposure to hypoxia.

Immunohistochemical staining for $\alpha$-SMC, a typical marker of SMCs, indicated that the hypoxia-induced thickening of the pulmonary arterioles was attributed to the increased numbers of SMCs, which was markedly reduced by HIF-1 $\alpha$ shRNA \#1 and \#2 (Fig. 2D). In normal lungs, only $11.1 \pm 4.4 \%$ of the pulmonary arterioles were fully muscularized, whereas exposure to chronic hypoxia significantly induced muscularization of the pulmonary arterioles $(31.8 \pm 7 \%$; $\mathrm{P}<0.001)$. Compared with the hypoxia group, pre-treatment with HIF-1 $\alpha$ shRNA \#1 significantly reduced the percentage of fully muscularized pulmonary arterioles $(19.1 \pm 4.5 \%$; $\mathrm{P}<0.01)$, whereas HIF- $1 \alpha$ shRNA\#2 reduced the muscularization of pulmonary arterioles to a lesser 
A

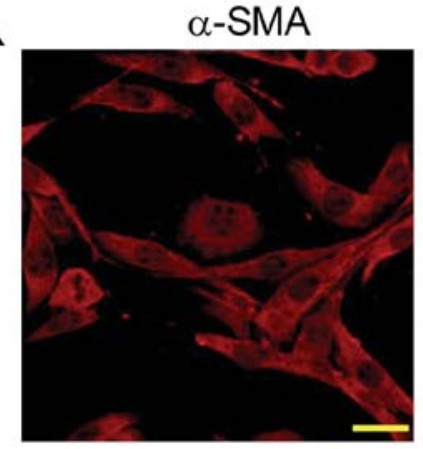

B

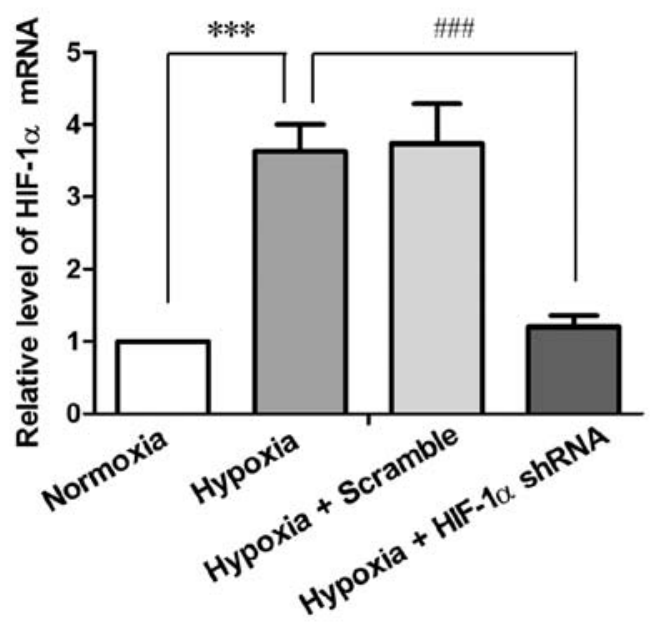

DAPI

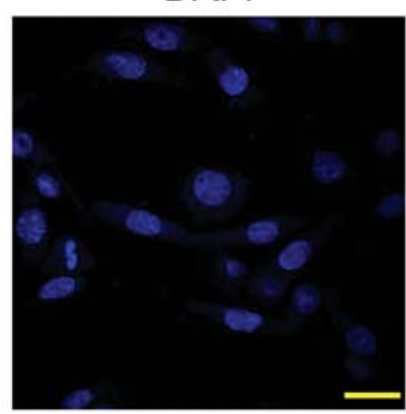

C

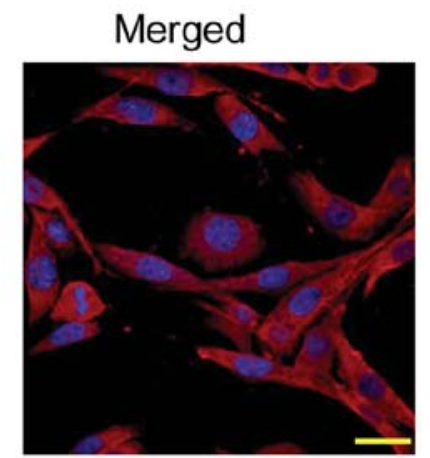

C
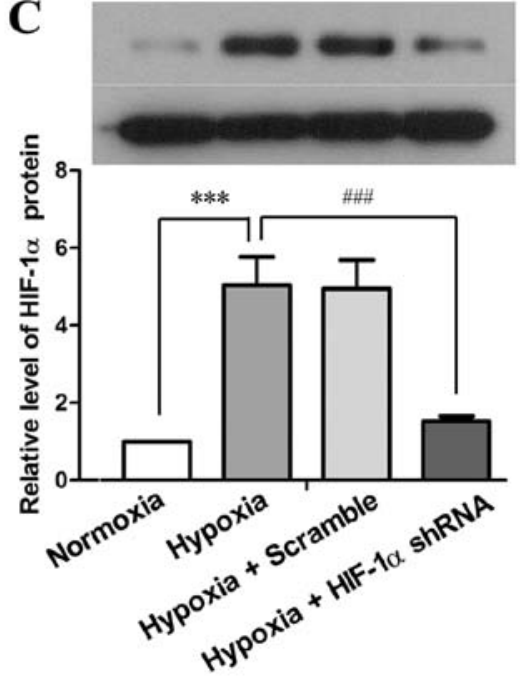

Figure 3. Hypoxia-inducible factor-1 $\alpha$ (HIF-1 $\alpha$ ) shRNA inhibits the hypoxia-induced upregulation of HIF-1 $\alpha$ in primary pulmonary artery smooth muscle cells (PASMCs). PASMCs were isolated from 6-8 weeks-old SD rats, and cultured in vitro. (A) Primary PASMCs were identified by the expression of $\alpha$-smooth muscle actin $(\alpha-$ SMA) using immunofluorescence staining. The cells were photographed under a fluorescence microscope under x600 magnification (scale bar, $20 \mu \mathrm{m})$. PASMCs were infected with lentiviruses harboring HIF-1 $\alpha$ shRNA \#1 or scramble shRNA prior to exposure to hypoxia for $48 \mathrm{~h}\left(5 \% \mathrm{O}_{2}\right)$, and the levels of (B) HIF-1 $\alpha$ mRNA and (C) HIF-1 $\alpha$ protein were assessed by RT-qPCR and western blot analysis, respectively. The figure shows the representative images from 3 independent experiments, and the values are expressed as the means $\pm \mathrm{SD}$ of triplicate experiments. ${ }^{* * *} \mathrm{P}<0.001$, hypoxia vs. normoxia; ${ }^{\# \# \prime} \mathrm{P}<0.001$, hypoxia + HIF-1 $\alpha$ shRNA vs. hypoxia.

extent $(23.6 \pm 5.3 \%)$ (Fig. 2E). These results demonstrated that the suppression of HIF-1 $\alpha$ expression by RNAi attenuated the symptoms of hypoxia-induced $\mathrm{PH}$ and pulmonary vascular remodeling.

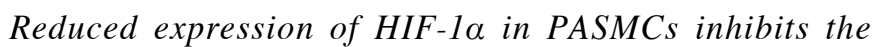
hypoxia-induced acceleration of cell proliferation and cell cycle progression. The deregulated proliferation of PAECs is regarded to play a central role in hypoxia-induced $\mathrm{PH}$ and vascular remodeling as vascular endothelial growth factor (VEGF) has been demonstrated to be a HIF-1 target that drives vascular remodeling (21), and PAEC-secreted factors can also promote PASMC proliferation (6,7). In this study, we focused on the endogenous role of HIF-1 $\alpha$ in PASMCs. PASMCs were prepared from 6-8-week-old SD rats, and the purity of the isolated cells was determined by immunofluorescence staining using a specific antibody against $\alpha$-SMC (Fig. 3A). Primary PASMCs were cultured in vitro, and infected with lentiviruses carrying HIF- $1 \alpha$ shRNA \#1 or scramble shRNA. Exposure to hypoxia $\left(5 \% \mathrm{O}_{2}\right)$ for $48 \mathrm{~h}$ resulted in a 2.63 -fold elevation of HIF-1 $\alpha$ mRNA expression $(\mathrm{P}<0.001)$ and in a 4.05-fold elevation of HIF-1 $\alpha$ protein expression $(\mathrm{P}<0.001)$ in the PASMCs compared with the cells under normoxic conditions (Fig. 3B and C). By contrast, HIF-1 $\alpha$ shRNA reduced the hypoxia-induced elevation of HIF-1 $\alpha$ by $85-90 \%$ at the mRNA and protein level $(\mathrm{P}<0.001)$. These results indicated a high efficiency of the HIF-1 $\alpha$ shRNA-mediated inhibition of HIF- $1 \alpha$ expression in PASMCs under hypoxic conditions.

The proliferation and cell cycle of the PASMCs treated with or without RNAi and exposed to hypoxia were assessed. As shown in Fig. 4A, $48 \mathrm{~h}$ of exposure to hypoxia doubled the number of PASMCs compared with those under normoxic conditions $(\mathrm{P}<0.001)$, while HIF-1 $\alpha$ shRNA markedly suppressed the hypoxia-accelerated proliferation of the PASMCs $(\mathrm{P}<0.001)$. Cell cycle analysis revealed that hypoxia disrupted the progression of the cell cycle of PASMCs, as indicated by the reduced number of cells in the $G_{0} /$ $G_{1}$ phase $(P<0.001)$ and the accumulation of the cells in the $\mathrm{G} 2$ phase $(\mathrm{P}<0.001)$ in the hypoxia-exposed cells as compared to the cells under normoxic conditions (Fig. 2B and C). This result implied hypoxia induced accelerated cell cycle and enhanced proliferation of PASMCs. On the contrary, treatment with HIF-1 $\alpha$ shRNA significantly attenuated the hypoxia-induced disruption of the cell cycle distribution at both the $G_{0} / G_{1}$ phase $(P<0.01)$ and $G 2$ phase $(P<0.001)$ in the PASMCs. These data suggest that the proliferation and cell cycle of PASMCs are directly regulated by HIF-1 $\alpha$ in response to hypoxia. 

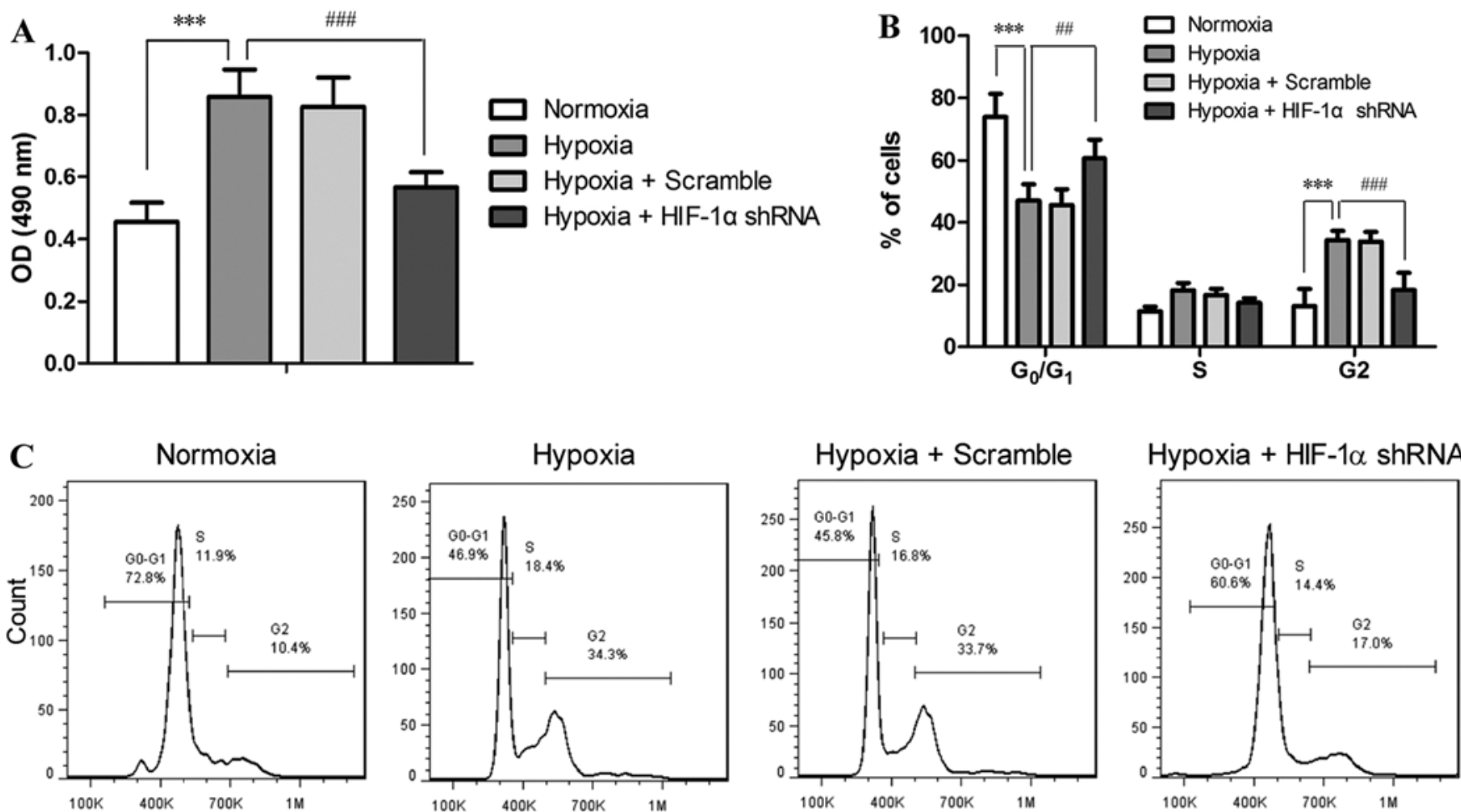

Figure 4. Hypoxia-inducible factor-1 $\alpha$ (HIF-1 $\alpha$ ) shRNA suppresses the hypoxia-induced acceleration of proliferation and cell cycle of pulmonary artery smooth muscle cells (PASMCs). PASMCs were infected with lentiviruses harboring HIF-1 $\alpha$ shRNA \#1 or scramble shRNA, and exposed to hypoxia (5\% $\mathrm{O}_{2}$ ) for $48 \mathrm{~h}$. (A) The number of viable cells was determined by MTT assay. (B and C) The cells were fixed and stained with PI, and cell cycle was analyzed by flow cytometry. (B) Statistical analysis of 3 independent experiments, and (C) a set of representative flow cytometry results. The values are expressed as the means \pm SD of triplicate experiments. ${ }^{* * *} \mathrm{P}<0.001$, hypoxia vs. normoxia; ${ }^{\# \prime} \mathrm{P}<0.01$ and ${ }^{\# \# \prime} \mathrm{P}<0.001$, hypoxia + HIF-1 $\alpha$ shRNA vs. hypoxia.

\section{Discussion}

HIF-1 $\alpha$ has been demonstrated to play an essential role in the pathophysiology of chronic hypoxia-induced $\mathrm{PH}$ in a mice with the heterozygous deletion of HIF-1 $\alpha$ (11) and in mice with the smooth muscle-specific disruption of HIF-1 $\alpha$ (12). Recently, HIF-1 $\alpha$ has been implicated in several molecular mechanisms accounting for the pathogenesis of $\mathrm{PH}$ (22-24). In addition, the increased expression of HIF-1 $\alpha$ has been found in the pulmonary arteries of many patients with idiopathic PH $(13,25)$. Although HIF-1 $\alpha$ is recognized to be a key player in PH, surprisingly, the potential of HIF- $1 \alpha$ as a therapeutic target in the treatment of PH has seldom been evaluated in previous studies. In this study, we employed a lentivirus-mediated gene delivery approach to introduce HIF-1 $\alpha$ shRNA to the pulmonary vessels in SD rats, and found that the suppression of the expression of HIF-1 $\alpha$ in the pulmonary arteries effectively attenuated chronic hypoxiainduced $\mathrm{PH}$ and pulmonary vascular remodeling. In addition, the reduction of HIF-1 $\alpha$ expression in primary PASMCs by RNAi significantly inhibited the hypoxia-induced acceleration of the proliferation and cell cycle of PASMCs, suggesting that the HIF-1 $\alpha$-mediated over-proliferation of PASMCs under hypoxic conditions may play an important part in the development of hypoxic $\mathrm{PH}$.

The protein level HIF-1 $\alpha$ is highly regulated by intracellular $\mathrm{O}_{2}$ tension. Under normoxic conditions, HIF- $1 \alpha$ is continuously synthesized and expressed in the cytoplasm, but is rapidly degraded by the ubiquitin-mediated pathway. However, under hypoxic conditions, the low availability of $\mathrm{O}_{2}$ inhibits the hydroxylation of HIF- $1 \alpha$ and the subsequent ubiquitination and proteasomal degradation, resulting in the accumulation of HIF-1 $\alpha$ protein in the nucleus and the initiation of the transcription of hypoxic responsive genes (26). In addition, the level of HIF-1 $\alpha$ is also regulated at the transcriptional level in response to a variety of growth factors via the activation of various signaling pathways, such as the phosphatidylinositol 3-kinase (PI3K) pathway or mitogen-activated protein kinase (MAPK) pathway $(27,28)$. In this study, exposure to chronic hypoxia resulted in the elevation of HIF-1 $\alpha$ mRNA and protein levels in the pulmonary arteries of SD rats and in the in vitro cultured PASMCs, suggesting that, in addition to the instant protein stabilization in the absence of $\mathrm{O}_{2}$, HIF-1 $\alpha$ expression is enhanced at the transcriptional level as an adaptive response to chronic hypoxia. Thus, the inhibition of HIF-1 $\alpha$ expression by RNAi is apparently an efficient approach to delay or alleviate hypoxia-induced pathological alterations.

As a critical feature of pulmonary vascular remodeling, the hypertrophy and hyperplasia of PASMCs contributed greatly to the sustained increase of pulmonary vascular resistance and pulmonary artery pressure in $\mathrm{PH}$ (20). In this study, we observed thatthe lentivirus-mediated delivery of HIF-1 $\alpha$ shRNA effectively inhibited HIF-1 $\alpha$ expression in the pulmonary arteries of SD rats that were exposed to chronic hypoxia, and such a reduction in HIF-1 $\alpha$ expression markedly attenuated the hypoxia-induced hyperplasia of PASMCs and the elevation of RVSP, an indicator of pulmonary arterial pressure. These results suggest a promising therapeutic potential of HIF-1 $\alpha$ shRNA for hypoxic PH.

Previous studies have demonstrated that PAECs can regulate PASMC proliferation by releasing growth stimulators, 
such as serotonin and fibroblast growth factor-2 (FGF-2) $(6,7)$, or by reducing the production of factors that normally suppress PASMC proliferation, such as apelin (29). In this study, by culturing PASMCs in vitro, we demonstrated that hypoxia induced the acceleration of PASMC proliferation independent of PAECs, but via HIF-1 $\alpha$-mediated hypoxic responses, suggesting that HIF- $1 \alpha$ plays a critical role in PASMC hyperplasia in hypoxia-induced $\mathrm{PH}$. These in vitro findings also support the observation in the mice with $\mathrm{PH}$ with smooth muscle-specific deletion of HIF-1 $\alpha$ that HIF-1 $\alpha$ in the smooth muscle cells contributes to the development of $\mathrm{PH}$ in chronic hypoxia (12). In addition to hypoxia, HIF-1 $\alpha$ has also been implicated in the growth factor-induced proliferation of PASMCs, presumably through the HIF-1-dependent expression of cyclin A $(30,31)$, which may be also involved in the accelerated cell cycle of PASMCs under chronic hypoxic conditions in this study.

Our in vivo study demonstrated that the intratracheal instillation of lentivirus-carried HIF- $1 \alpha$ shRNA effectively attenuated the hypoxia-induced elevation of RVSP, pulmonary vascular remodeling and the muscularization of arterioles, providing evidence for the therapeutic potential of HIF-1 $\alpha$ shRNA for hypoxic PH. Genetic medicine has long been proposed to be of tremendous therapeutic potential and has been assessed in several diseases $(32,33)$. One major issue associated with the virus-based gene transduction system is the host immune response, thus non-viral delivery methods with satisfactory efficiency are being developed for potential clinical applications (34). Hence, the efficiency of non-virusbased delivery of HIF-1 $\alpha$ shRNA to pulmonary arteries and its efficacy for the treatment of $\mathrm{PH}$ is to be evaluated in future studies for the potential use of HIF- $1 \alpha$ shRNA in the treatment of PH.

In conclusion, this study demonstrated that the inhibition of HIF-1 $\alpha$ expression by the lentivirus-mediated delivery of HIF-1 $\alpha$ shRNA into rat pulmonary arteries effectively attenuated the symptoms associated with hypoxia-induced $\mathrm{PH}$, which was, at least partially, attributed to the suppression of the hypoxia-induced hyperplasia of PASMCs when HIF-1 $\alpha$ was silenced. Moreover, this study provides the preliminary evidence for the therapeutic potential of HIF-1 $\alpha$ shRNA in the treatment of hypoxic $\mathrm{PH}$.

\section{References}

1. Simonneau G, Gatzoulis MA, Adatia I, Celermajer D, Denton C, Ghofrani A, Gomez Sanchez MA, Krishna Kumar R, Landzberg M, Machado RF, et al: Updated clinical classification of pulmonary hypertension. J Am Coll Cardiol 62 (Suppl 25): D34-D41, 2013.

2. D'Alonzo GE, Barst RJ, Ayres SM, Bergofsky EH, Brundage BH, Detre KM, Fishman AP, Goldring RM, Groves BM, Kernis JT, et al: Survival in patients with primary pulmonary hypertension. Results from a national prospective registry. Ann Intern Med 115: 343-349, 1991 .

3. Humbert M, Sitbon O, Chaouat A, Bertocchi M, Habib G, Gressin V, Yaïci A, Weitzenblum E, Cordier JF, Chabot F, et al: Survival in patients with idiopathic, familial, and anorexigenassociated pulmonary arterial hypertension in the modern management era. Circulation 122: 156-163, 2010.

4. Shimoda LA and Semenza GL: HIF and the lung: Role of hypoxia-inducible factors in pulmonary development and disease. Am J Respir Crit Care Med 183: 152-156, 2011.

5. Stenmark KR, Fagan KA and Frid MG: Hypoxia-induced pulmonary vascular remodeling: Cellular and molecular mechanisms. Circ Res 99: 675-691, 2006.
6. Thompson $\mathrm{K}$ and Rabinovitch M: Exogenous leukocyte and endogenous elastases can mediate mitogenic activity in pulmonary artery smooth muscle cells by release of extracellular-matrix bound basic fibroblast growth factor. J Cell Physiol 166: 495-505, 1996.

7. Dewachter L, Adnot S, Fadel E, Humbert M, Maitre B Barlier-Mur AM, Simonneau G, Hamon M, Naeije R and Eddahibi S: Angiopoietin/Tie2 pathway influences smooth muscle hyperplasia in idiopathic pulmonary hypertension. Am J Respir Crit Care Med 174: 1025-1033, 2006.

8. Jones PL, Cowan KN and Rabinovitch M: Tenascin-C, proliferation and subendothelial fibronectin in progressive pulmonary vascular disease. Am J Pathol 150: 1349-1360, 1997.

9. Wang GL, Jiang BH, Rue EA and Semenza GL: Hypoxiainducible factor 1 is a basic-helix-loop-helix-PAS heterodimer regulated by cellular $\mathrm{O}_{2}$ tension. Proc Natl Acad Sci USA 92: 5510-5514, 1995.

10. Semenza GL: Targeting HIF-1 for cancer therapy. Nat Rev Cancer 3: 721-732, 2003.

11. Yu AY, Shimoda LA, Iyer NV, Huso DL, Sun X, McWilliams R, Beaty T, Sham JS, Wiener CM, Sylvester JT and Semenza GL: Impaired physiological responses to chronic hypoxia in mice partially deficient for hypoxia-inducible factor lalpha. J Clin Invest 103: 691-696, 1999.

12. Ball MK, Waypa GB, Mungai PT, Nielsen JM, Czech L, Dudley VJ, Beussink L, Dettman RW, Berkelhamer SK, Steinhorn RH, et al: Regulation of hypoxia-induced pulmonary hypertension by vascular smooth muscle hypoxia-inducible factor-1 $\alpha$. Am J Respir Crit Care Med 189: 314-324, 2014.

13. Tuder RM, Chacon M, Alger L, Wang J, Taraseviciene-Stewart L, Kasahara Y, Cool CD, Bishop AE, Geraci M, Semenza GL, et al: Expression of angiogenesis-related molecules in plexiform lesions in severe pulmonary hypertension: Evidence for a process of disordered angiogenesis. J Pathol 195: 367-374, 2001.

14. Brusselmans K, Compernolle V, Tjwa M, Wiesener MS, Maxwell PH, Collen D and Carmeliet P: Heterozygous deficiency of hypoxia-inducible factor-2alpha protects mice against pulmonary hypertension and right ventricular dysfunction during prolonged hypoxia. J Clin Invest 111: 1519-1527, 2003.

15. Zhang R, Shi L, Zhou L, Zhang G, Wu X, Shao F, Ma G and Ying K: Transgelin as a therapeutic target to prevent hypoxic pulmonary hypertension. Am J Physiol Lung Cell Mol Physiol 306: L574L583, 2014.

16. Wang L, Zhou Y, Li M and Zhu Y: Expression of hypoxiainducible factor-1 $\alpha$, endothelin-1 and adrenomedullin in newborn rats with hypoxia-induced pulmonary hypertension. Exp Ther Med 8: 335-339, 2014

17. Ko EA, Song MY, Donthamsetty R, Makino A and Yuan JX: Tension measurement in isolated rat and mouse pulmonary artery. Drug Discov Today Dis Models 7: 123-130, 2010.

18. Christou H, Morita T, Hsieh CM, Koike H, Arkonac B, Perrella MA and Kourembanas S: Prevention of hypoxia-induced pulmonary hypertension by enhancement of endogenous heme oxygenase-1 in the rat. Circ Res 86: 1224-1229, 2000.

19. Sreejayan $\mathrm{N}$ and Yang X: Isolation and functional studies of rat aortic smooth muscle cells. Methods Mol Med 139: 283-292, 2007.

20. Jones R, Zapol WM and Reid L: Pulmonary artery remodeling and pulmonary hypertension after exposure to hyperoxia for 7 days. A morphometric and hemodynamic study. Am J Pathol 117: 273-285, 1984.

21. Hänze J, Weissmann N, Grimminger F, Seeger W and Rose F: Cellular and molecular mechanisms of hypoxia-inducible factor driven vascular remodeling. Thromb Haemost 97: 774-787, 2007.

22. Fukai K, Nakamura A, Hoshino A, Nakanishi N, Okawa Y, Ariyoshi M, Kaimoto S, Uchihashi M, Ono K, Tateishi S, et al: Pyk2 aggravates hypoxia-induced pulmonary hypertension by activating HIF-1 $\alpha$. Am J Physiol Heart Circ Physiol 308: H951-H959, 2015.

23. Shan F, Li J and Huang QY: HIF-1 alpha-induced upregulation of miR-9 contributes to phenotypic modulation in pulmonary artery smooth muscle cells during hypoxia. J Cell Physiol 229: 1511-1520, 2014.

24. Chettimada S, Gupte R, Rawat D, Gebb SA, McMurtry IF and Gupte SA: Hypoxia-induced glucose-6-phosphate dehydrogenase overexpression and -activation in pulmonary artery smooth muscle cells: Implication in pulmonary hypertension. Am J Physiol Lung Cell Mol Physiol 308: L287-L300, 2015.

25. Fijalkowska I, Xu W, Comhair SA, Janocha AJ, Mavrakis LA, Krishnamachary B, Zhen L, Mao T, Richter A, Erzurum SC and Tuder RM: Hypoxia inducible-factorlalpha regulates the metabolic shift of pulmonary hypertensive endothelial cells. Am J Pathol 176: 1130-1138, 2010. 
26. Maxwell PH, Wiesener MS, Chang GW, Clifford SC, Vaux EC, Cockman ME, Wykoff CC, Pugh CW, Maher ER and Ratcliffe PJ: The tumour suppressor protein VHL targets hypoxia-inducible factors for oxygen-dependent proteolysis. Nature 399: 271-275, 1999.

27. Fukuda R, Hirota K, Fan F, Jung YD, Ellis LM and Semenza GL: Insulin-like growth factor 1 induces hypoxia-inducible factor 1-mediated vascular endothelial growth factor expression, which is dependent on MAP kinase and phosphatidylinositol 3-kinase signaling in colon cancer cells. J Biol Chem 277: 38205-38211, 2002.

28. Zhong H, Chiles K, Feldser D, Laughner E, Hanrahan C, Georgescu MM, Simons JW and Semenza GL: Modulation of hypoxia-inducible factor 1alpha expression by the epidermal growth factor/phosphatidylinositol 3-kinase/PTEN/AKT/FRAP pathway in human prostate cancer cells: Implications for tumor angiogenesis and therapeutics. Cancer Res 60: 1541-1545, 2000.

29. Alastalo TP, Li M, Perez VJ, Pham D, Sawada H, Wang JK, Koskenvuo M, Wang L, Freeman BA, Chang HY and Rabinovitch M: Disruption of PPAR $\gamma / \beta$-catenin-mediated regulation of apelin impairs BMP-induced mouse and human pulmonary arterial EC survival. J Clin Invest 121: 3735-3746, 2011.
30. Schultz K, Fanburg BL and Beasley D: Hypoxia and hypoxiainducible factor-1alpha promote growth factor-induced proliferation of human vascular smooth muscle cells. Am J Physiol Heart Circ Physiol 290: H2528-H2534, 2006.

31. Imanishi $M$, Tomita S, Ishizawa K, Kihira Y, Ueno $M$, Izawa-Ishizawa Y, Ikeda Y, Yamano N, Tsuchiya K and Tamaki T: Smooth muscle cell-specific Hif-1 $\alpha$ deficiency suppresses angiotensin II-induced vascular remodelling in mice. Cardiovasc Res 102: 460-468, 2014.

32. Clayman GL, el-Naggar AK, Lippman SM, Henderson YC, Frederick M, Merritt JA, Zumstein LA, Timmons TM, Liu TJ, Ginsberg L, et al: Adenovirus-mediated p53 gene transfer in patients with advanced recurrent head and neck squamous cell carcinoma. J Clin Oncol 16: 2221-2232, 1998.

33. Manno CS, Pierce GF, Arruda VR, Glader B, Ragni M, Rasko JJ, Ozelo MC, Hoots K, Blatt P, Konkle B, et al: Successful transduction of liver in hemophilia by AAV-Factor IX and limitations imposed by the host immune response. Nat Med 12: 342-347, 2006.

34. Kim SS, Garg H, Joshi A and Manjunath N: Strategies for targeted nonviral delivery of siRNAs in vivo. Trends Mol Med 15: 491-500, 2009. 